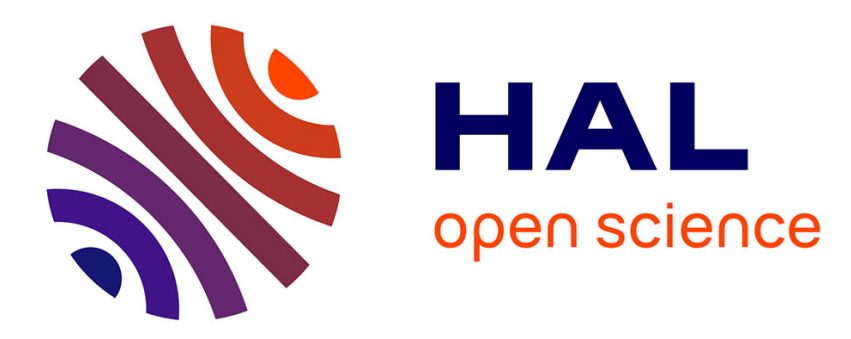

\title{
Antiviral escape strategies developed by bunyaviruses pathogenic for humans
}

Nicolas Le May, Michele Bouloy

\section{To cite this version:}

Nicolas Le May, Michele Bouloy. Antiviral escape strategies developed by bunyaviruses pathogenic for humans. Frontiers in Bioscience, 2012, S4 (3), pp.1065-1077. 10.2741/s318 . inserm-02462953

\section{HAL Id: inserm-02462953 https://www.hal.inserm.fr/inserm-02462953}

Submitted on 31 Jan 2020

HAL is a multi-disciplinary open access archive for the deposit and dissemination of scientific research documents, whether they are published or not. The documents may come from teaching and research institutions in France or abroad, or from public or private research centers.
L'archive ouverte pluridisciplinaire HAL, est destinée au dépôt et à la diffusion de documents scientifiques de niveau recherche, publiés ou non, émanant des établissements d'enseignement et de recherche français ou étrangers, des laboratoires publics ou privés. 


\title{
Antiviral escape strategies developed by bunyaviruses pathogenic for humans
}

\author{
Nicolas Le May ${ }^{1}$, Michele Bouloy ${ }^{2}$ \\ ${ }^{I}$ Institut de Génétique et de Biologie Moléculaire et Cellulaire, CNRS/INSERM/ULP, BP 163, 67404 Illkirch Cedex, C. U. \\ Strasbourg, France, ${ }^{2}$ Institut Pasteur, Unite de Génétique Moléculaire des Bunyavirus, 25 rue du Dr Roux, Paris, France
}

\section{TABLE OF CONTENTS}

1. Abstract

2. Introduction

3. The innate immunity

4. Orthobunyaviruses

4.1. Bunyamwera Virus

4.2. La Crosse Virus

5. Hantaviruses

5.1. Glycoproteins Gn and Gc

5.2. Nucleocapsid

5.3. NSs proteins

6. Nairoviruses: Crimea-Congo Hemorrhagic Fever Virus

7. Phleboviruses: Rift Valley Fever Virus

7.1. The multifunctionality of the RVFV NSs protein

8. Perspective

7.2. Anti-apoptotic role of RFVF NSm proteins

9. Acknowledgements

10. References

\section{ABSTRACT}

New or re-emerging pathogens for humans have emerged outside of their usual endemic range during the last decade originating severe public health concern and economical losses. Climate changes have played a significant role in the emergence or re-emergence of arboviruses. Among these pathogens, several viruses belong to the Bunyaviridae family. This family is composed of RNA viruses grouped into five genera-Orthobunyavirus, Hantavirus, Nairovirus, Phlebovirus and Tospovirus characterized by their antigenic, genetic and ecological properties. These viruses use cellular proteins to promote their own replication/transcription and reciprocally the host induces, in response, an important transcriptional reprogramming to activate antiviral defences including the interferon type I pathways. The virulence of the pathogenic bunyaviruses is directly linked to the roles of viral virulence factors and their capacity to counteract the host pathways. This review summarizes the various strategies developed by the different genera of the Bunyaviridae family to overcome and escape the innate immune response and eventually other cellular functions.

\section{INTRODUCTION}

During the past decade, the emergence of either emerging or re-emerging virus diseases in new areas of the world occurred with increasing frequency and became a serious public health concern and economical losses. Arboviruses are distributed worldwide and represent approximately $30 \%$ of all emerging infectious diseases during the last decade (1). Viruses such as West Nile (WNV), Chikungunya (CHIKV), Dengue (DENV), Yellow Fever (YFV), Crimea-Congo Hemorrhagic Fever $(\mathrm{CCHF})$ and Rift Valley Fever (RVFV) viruses, which are pathogenic for humans and/or animals have emerged outside of their usual endemic range and caused epidemics in North America, Europe and the Arabian Peninsula. Their emergence may be related to the climate fluctuations due to the global warming and/or human activities (human travel increasing, deforestation, political and military activities) that facilitate the dispersion of the arthropods beyond their current geographic boundaries $(2,3)$. As an example of new emerging pathogens, one should recall the newly identified phlebovirus isolated in China. It is transmitted by ticks and closely related to the tick borne Uukuniemi (UUKV) virus. However, in contrast with UUKV, which is not pathogenic for humans, this novel phlebovirus designated severe fever with thrombocytopenia syndrome virus or Huaiyangshan virus depending on the laboratory where it was isolated, is responsible for severe thrombocytosis and multi-organ dysfunction with high morbidity and mortality (initial fatality rate 30\%) $(4,5)$. Studies on these arboviruses thus become a major issue since neither safe vaccine for protection nor antiviral treatments for therapy is currently available. Among these emerging pathogens, several viruses belong to the large family of RNA viruses, the Bunyaviridae family (Table 1).

The Bunyaviridae family comprises more than 350 members and is subdivided in five genera (i.e. Orthobunyavirus, Hantavirus, Nairovirus, Phlebovirus and Tospovirus) characterized by their antigenic, genetic and ecological properties. Orthobunyaviruses, nairoviruses and phleboviruses infect vertebrates and are vectored by haematophagous arthropods including mosquitoes, ticks, midges and sandflies whereas tospoviruses are plant pathogens and are vectored by different thrips (6). In 
contrast, hantaviruses are not transmitted by arthropods but by rodents and insectivores which act as reservoirs. Infections by hantaviruses are persistent in their reservoir hosts and humans become infected through contamination by excretions or carcasses of infected reservoirs (7).

All the members of this family, referred as bunyaviruses, are enveloped, spherical virions between $80-120 \mathrm{~nm}$ in diameter with its replicative cycle in the cytoplasm and maturation and budding of the newly formed particles in the Golgi apparatus $(8,9)$. These viruses possess single-stranded RNA genomes that consist of three segments L (Large), M (Medium) and $\mathrm{S}$ (Small) having a negative- or ambi-sense polarity (6). The L and M segments code respectively for the viral RNA-dependent RNA polymerase (L-RdRp) and a precursor to the envelope glycoproteins ( $\mathrm{Gn}$ and $\mathrm{Gc}$ ). The $\mathrm{S}$ segment codes for the internal protein $\mathrm{N}$ or nucleocapsid protein that is able to oligomerize and associate with the viral polymerase and the three different segments of the viral genome to form viral ribonucleoparticles (RNPs) packaged into virions. Depending on the genus, other proteins are encoded by the $\mathrm{M}$ and $\mathrm{S}$ segments, namely the non-structural proteins NSm1, NSm2 and NSs harbouring multiple roles. The virulence of the pathogenic bunyaviruses illustrates the permanent co-evolution between the viruses and their hosts. The virus utilizes cellular proteins or functions to promote their own replication/transcription. This is particularly well illustrated in the case of bunyaviruses which utilize capped oligonucleotides from host cell mRNAs to prime transcription through the capsnatching mechanism mediated by the L-RdRp which possesses an endonuclease activity to cleave the capped oligonucleotides (10). In response to viral infection, the host induces an important transcriptional reprogramming to activate various defences including the innate immunity. However, viruses have developed strategies to overcome the numerous host responses; this will be described in the following sections of this review.

\section{THE INNATE IMMUNITY}

In many cases, arboviruses infect vertebrates through mosquito or tick bites, which inoculate the virus in the skin, making Dendritic Cells (DCs) such as skin Langerhans cells the primary cell targets $(11,12)$. Infected Langerhans cells migrate to the draining lymph node, allowing the virus to enter the bloodstream and disseminate. After pathogen intrusion, the first response of the cell or the organism is an inflammatory reaction including secretion of soluble mediators such as cytokines and chemokines. Among these mediators, Interferons (IFNs) and especially type I IFNs (IFN $\alpha / \beta$ ) are the most efficient to mount a rapid host response with the aim to block the viral replication $(13,14)$. The initial response to infection involves the production and secretion of IFN $\alpha 1$ and IFN $\beta$ followed by a subsequent amplification phase linked to induction of other IFN $\alpha$ subtypes. The secreted IFNs act in autocrine and paracrine manner to stimulate the expression of antiviral, antiproliferative and immunomodulatory genes.

The induction of type I IFNs occurs mainly by an intracellular pathway and necessitates the initial recognition between specific cellular Pattern Recognition Receptors (PRR) and viral inducers so-called Pathogen-Associated Molecular Patterns (PAMP). Double-stranded (ds) RNA or 5'-triphosphorylated single-stranded (ss) RNA from RNA viruses trigger several cytosolic PAMPs including two RNA helicases, RIG-I and MDA-5, and the dsRNA-dependent Protein Kinase R (PKR). The binding of viral RNA to RIG-I and MDA-5 induces a signaling chain, which leads to the activation and nuclear translocation of several transcription factors including the constitutive Interferon Regulatory Factor-3 (IRF3) and NF- $\mathrm{BB}$. The RIG-I and MDA-5 dependent pathways result in the phosphorylation of IRF-3 by TBK1, the homodimerisation and nuclear translocation of IRF-3 being a prerequisite to initiate the messenger RNA (mRNA) IFN $\beta$ and human IFN $\alpha 1$ synthesis $(15,16)$. The kinase PKR is a constitutive protein that is activated by dsRNA inducing its auto-phosphorylation. The activated PKR can therefore

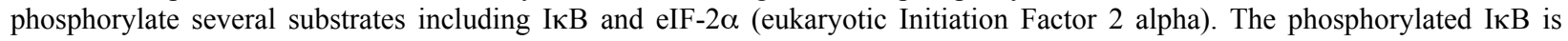
degraded through the proteasome activating the transcription factor NF- $\mathrm{BB}$ whereas its effect on eIF-2 $\alpha$ leads to the inhibition of translation of cellular and viral mRNA.

The secreted IFN $\alpha 1$ and IFN $\beta$, triggered through the IRF-3-dependent induction, are able to bind and activate IFNAR1 and IFNAR2 present on all host cells. These IFNARs dimerize and induce the phosphorylation of members of Janus Kinase (JAK), TYK-2 and JAK1 that are pre-associated with their cytoplasmic tails. The latent cytoplasmic transcription factors from the Signal Transducer and Activator of Transcription (STAT) family are therefore phosphorylated by JAK1 and TYK-2 leading to their activation $(17,18)$. Phosphorylated STAT1 and STAT2 heterodimerize and recruit IRF-9 to form a complex called IFN stimulated gene factor 3 (ISGF-3) that translocates to the nucleus, binds to IFN-stimulated response elements (ISRE) localized in numerous IFN-induced gene promoters such as IFN-Stimulated Genes (ISGs) and IRF-7 and activates the transcription of these genes (Figure 1). IRF-7 is not constitutively expressed in most cells (except in plasmacytoid Dendritic Cells, pDCs) and is an IFN-inducible and virus-inducible protein similar to IRF-3. Active IRF-7 homodimers or heterodimers with IRF-3, bind to promoter of all IFN $\alpha$ genes, and are therefore responsible for the induction of delayed type I IFNs and amplification of the IFN response (16) (Figure 1). IFNs also induce more than 350 ISGs, which have antiviral, antimicrobial, antiproliferative and immunomodulatory functions (19). The proteins induced by IFNs include enzymes, signalling proteins, chemokines, cytokines, transcription factors, heat shock proteins, surface glycoproteins or pro-apoptotic proteins. Among this list, only a few ones have been characterized with antiviral activities such as the Mx GTPases, PKR, the 2',5' Oligoadenylate Synthetases (2-5 OAS)/RNAse L system, the RNA-specific adenosine deaminase 1 (ADAR1), viperin, ISG20 or p56 which are largely described in more detailed in other reviews (20). In response to the type I IFNs action, each virus targets specific cellular proteins and 
usually possesses unique strategy to counteract these pathways. This review illustrates the diversity and originality in the strategies developed by different pathogenic bunyaviruses.

\section{ORTHOBUNYAVIRUSES}

This genus consists of more than 150 viruses; it is subdivided into 19 different serogroups: among which the California, Bunyamwera and Simbu serogroups comprise respectively, three serious human pathogens: La Crosse, Ngari and Oropouche viruses. Bunyamwera virus is the prototype for the Orthobunyavirus genus and the Bunyaviridae family. The orthobunyaviruses are able to replicate both in mammalians and in insects. The infection in mammalian cells is lytic and causes host cell shut off and cell death whereas it is noncytolytic and leads to viral persistent in invertebrate cells. Besides the classical organization, the M segment encodes a polyprotein precursor post-translationally cleaved into the envelope glycoproteins Gn and Gc and a third protein NSm of unknown function. In addition to the nucleocapsid, the S segment also encodes the NSs protein in an overlapping reading frame. The NSs protein has been reported to act as an interferon antagonist playing an important role in viral pathogenesis (21-23). The innate immune escape mediated by NSs protein was more precisely studied for two orthobunyaviruses Bunyamwera (BUNV) and La Crosse (LACV) viruses. The NSs protein impairs the antiviral response by preventing cellular transcription through the proteolysis of RNA polymerase II (RNAPII) (Figure 2).

\subsection{Bunyamwera virus}

The BUNV NSs protein is predominantly cytoplasmic although a significant fraction was also observed in the nucleus of cells transfected with a Flag-NSs expressing plasmid (22). Upon infection with wild type (wt) BUNV, the induction of IFN or IRF-3 mediated pro-apoptosis genes is abolished although the activation of IRF-3 and PKR is maintained indicating the NSs effect is downstream the primary signaling chain of innate immunity $(24,25)$. In BUNV-infected mammalian cells, NSs inhibits the phosphorylation of serine 2 in heptapeptide repeat of the CTD of RNA polymerase II and induces also its degradation (26, 27). Such a dysregulation of the host transcription machinery results from the interaction between the C-terminal region of NSs and Med8, a subunit of mediator complex (27). Mediator regulates RNAPII phosphorylation CTD-ser-5 residue and contacts the CTD-ser-2 kinase P-TEFb (28, 29). Moreover, Med8 can form an ubiquitin E3 ligase with Elongin B/C, Cul2 and Rbx1 targeting putatively RNAPII. Thus, NSs can evade the innate immune response by a general block of transcription of all cellular proteincoding genes including type I IFNs. This inhibition of the host transcription machinery caused by NSs is at least partially responsible for the shut off of cell protein synthesis $(23,26)$. Indeed, a recent study demonstrated the interaction with Med8 alone is not sufficient to impair the cellular gene expression and that other host partners requiring the N-terminal region of NSs are involved in this process (30).

\subsection{La Crosse virus}

LACV causes severe encephalitis and meningitis in children in the Midwestern United States including 300,000 cases per year with more than $10 \%$ developing long-lasting neurological defaults. LACV NSs protein was identified as a type I IFNs antagonist without affecting the PKR activity and the RIG-I pathway that is normally induced in infected cells $(21,31,32)$. Two studies have ascribed the LCAV NSs protein as RNAi suppressor and pro-apoptotic protein since it has similarities with the Reaper protein from Drosophila involved in translation inhibition and apoptosis in insects (33-35). However, these two putative functions seem to be side effects of the NSs molecular mechanisms to inhibit IFN induction. Indeed, expression of NSs has no apparent pro-apoptotic activity in insects and no advantage conferred by NSs was detected for the RNAi inhibition in insects and mammalian cells (21). A recent study indicates that NSs acts downstream the canonical RIG-I/IRF-3 signaling pathway and its effect is based on the shutdown of RNAPII-dependent transcription. LACV NSs exploits the response of cells to damaged DNA to induce the degradation of the elongating RNAPII. Indeed, DNA damage causes the arrest of elongating RNAPII, which in turn triggers the proteolysis through the ubiquitin/proteasome pathway. In LACV-infected cells, NSs activates part of the DDR machinery such as the DDR pak6 gene and the phosphorylation of H2A.X (32). The authors suggest that such an effect can lead to a general mRNA shut off. However the precise mechanism inhibiting and degrading the elongating RNAPII through LACV NSs remains to be characterized.

\section{HANTAVIRUSES}

Contrary to other bunyaviruses, hantaviruses are not transmitted by arthropods. They are rodent-borne viruses, present throughout the world. They are classified into New World or Old World based on their geographic location. Pathogenic New World hantaviruses (Andes, ANDV, Sin Nombre, SNV and New York1, NY-1V viruses) cause a Hantavirus Cardiopulmonary Syndrome (HCPS) in Americas whereas pathogenic Old World hantaviruses (Hantaan, HNTV, Puumala, PUUV and Seoul, SEOV viruses) cause a Hemorrhagic Fever with Renal Syndrome (HFRS) in Europe and Asia. The New World hantaviruses Andes and Sin Nombre are responsible for mortality rate of 50\% while the Old World hantavirus Hantaan can reach a mortality rate of $15 \%$ (7). Hantaviruses developed species-specific mechanisms to escape the innate immune responses. However, even non-pathogenic viruses (Tula TULV, Prospect Hill PHV viruses) antagonize some IFN pathways indicating that the IFN dysregulation alone is insufficient for hantaviruses to cause disease $(36,37)$. The different hantaviral strategies may involve several viral proteins including the glycoproteins, the nucleocapsid and the NSs proteins that can collectively block different steps in the type I IFNs pathways (Figure 3). 


\subsection{Glycoproteins Gn and Gc}

Two reports have demonstrated that the ectopic expression of the glycoprotein Gn cytoplasmic tail from NY-1V was sufficient to inhibit the NF-KB and IRF-3-directed transcriptional responses. The TBK1 and RIG-I-directed steps were involved in such dysregulation and especially the components of TBK1 complexes $(38,39)$. TBK1 plays a crucial role in the IFN regulation since it directs the activation of both IRF-3 and NF- $\kappa B(15,40)$. TRAF3 forms a complex with TBK1 and links upstream IFN signaling responses of the RIG-I/MDA-5 induced MAVS (IPS-1/Cardiff/VISA) to the TBK-1-directed phosphorylation of IRF-3 (41). TBK1 also activates NF-KB through interactions with TRAF2 (40). The NY-1V Gn cytoplasmic tail interacts with the TRAF3 N-terminus and consequently impairs cellular TBK-1-TRAF3 complex formation (39) (Figure 3). It was also suggested that such viral glycoprotein could block, through a similar mechanism, the TRAF2-directed NF- $\mathrm{KB}$ activation. For other pathogenic hantaviruses, both glycoproteins Gn and Gc could be necessary to overcome the IFN response. Indeed, the expression of the glycoprotein precursor (GPC) of SNV and ANDV is sufficient to inhibit the IFN $\beta$ induction, IRF-3 activation and the JAK/STAT signaling through the blockage of the phosphorylation and the nuclear translocation of STAT1/STAT2 $(36,42)$.

\subsection{Nucleocapsid}

The nucleocapsid is the most abundant viral protein in bunyavirus-infected cells. It is essential for the replicative cycle playing multiple functions. In the case of hantaviruses, it was shown to be involved in the formation of RNP through its homotrimerization and its interaction with viral RNA $(43,44)$. The nucleocapsid participates to the translation initiation mechanism through its interaction with Ribosomal Protein S19 (RPS19) (45). The IFN antagonism was also associated to this viral protein. Indeed, N proteins from PUUV, HTNV and TULV have been shown to interact respectively with the apoptotic protein Daxx, the Ubiquitin-conjugating enzyme 9 (Ubc9) and the Small Ubiquitin-like Modifier 1 (SUMO-1) (46-48). However the link between these interactions and the IFN escape remains to be determined. HTNV can also block the activation of NF- $\mathrm{KB}$ via the TNF- $\alpha$ through its nucleocapsid. It was demonstrated that the $\mathrm{N}$ protein interacts with importin $\alpha$ proteins, impairing the nuclear translocation of activated NF-KB that remains into the cytoplasm $(49,50)$. Another role linked to the nucleocapsid of ANDV is the inhibition of STAT1 phosphorylation, nuclear translocation and IFN $\beta$-induced ISRE activity. However, the ANDV $\mathrm{N}$ protein alone is not sufficient but rather functions in synergy with the glycoprotein precursor (GPC) to inhibit significantly these different steps of IFN signaling (36).

\subsection{NSs proteins}

The $\mathrm{S}$ segment of some hantaviruses possesses an additional open reading frame (ORF) coding for a non-structural protein NSs and overlapping the N ORF. This is a coding strategy resembling to the one observed for the orthobunyaviruses. Such an ORF is found in the S segments of hantaviruses transmitted by Arvicolinae and Sigmodontinae rodents (voles and lemmings of the north hemisphere and New world mice and rats) whereas it is absent for hantaviruses associated with Old World mice and rats (51). Recent studies have demonstrated that the NSs of TULV could accumulate in perinuclear area and was necessary for the viral survival in IFN-competent cells but not in IFN-deficient cells $(52,53)$. Moreover, the NSs protein of TULV and PUUV expressed via recombinant plasmids inhibited the induction of IFN $\beta$ and the activation of IRF-3 and NF- $\mathrm{KB}$ responsive promoters but to a weaker extent compared to the NSs protein from BUNV or RVFV (54). The host protein synthesis is not importantly impaired in TULV and PUUV-infected cells arguing for a weak effect of the NSs protein on cellular transcription. Contrary to the other bunyaviruses, hantaviruses have developed a different strategy to avoid more gently the IFN response. Indeed the hantaviruses are the only bunyaviruses causing an asymptomatic persistent infection in their rodent hosts (55). Such viruses encode several proteins antagonizing multiple cellular targets collectively inhibiting different steps of the antiviral response.

\section{NAIROVIRUSES: CRIMEA-CONGO HEMORRHAGIC FEVER VIRUS}

The Nairovirus genus includes 34 viruses grouped into seven different serotypes. All of the members are transmitted by argasid or ixodid ticks, but only three have been implicated as causes of human disease: the Dugbe (DUGV) and Nairobi sheep disease (NSD) and Crimean Congo hemorrhagic fever (CCHF) viruses, which is the most important human pathogen amongst them. CCHF is the causative agent of severe hemorrhagic fever in human transmitted either through bites by infected ticks or by nosocomial contamination (56). The average mortality rate is $30 \%$ but can reach $70 \%$ in some outbreaks. Mice lacking IFNAR or STAT1 are very sensitive to the CCHF infection, indicating the strong antiviral effect of type I IFNs (57, 58). Moreover, pretreatment with type I IFNs inhibits CCHF replication, involving the activity of ISGs like MxA, whereas an established CCHF infection is insensitive to subsequent treatment $(59,60)$. CCHF can counteract the IFN signaling through at least three independent mechanisms involving different viral elements. Firstly, the RIG-I dependent pathway is not activated since CCHFV does not produce significant amounts of the well-known inducers, which include the dsRNA and the 5' triphosphate ssRNA (61). It was proposed that CCHF cleaves the 5' triphosphate group during the replication to evade the recognition by this PRR. Secondly, it was demonstrated that the virus possesses at least one factor of virulence as an IFN antagonist that can delay the activation of IRF-3 (62). However, the factor is not identified yet. The existence of a coding capacity for a NSs-like gene in the S segment has been suggested but the protein remains to be determined. Thirdly, an ovarian tumor (OTU) domain has been identified on the L-RdRp of CCHF, DUGV and NSD (63). Proteins with an OTU domain belong to proteases super family deconjugating a broad spectrum of proteins modified with Ubiquitin (Ub) or ubiquitin-like proteins like IFN-Stimulated Gene 
product 15 (ISG15). The expression of a mutant L-RdRp defective in OTU protease function did not impair the replication and the transcription of a CCHF minigenome system indicating that the L-OTU activity is not required for these functions of L-RdRp (64). However, a role in the innate immunity escape has been suggested. The L-OTU activity would deconjugate ubiquitin and ISG15 from different cellular targets dysregulating their activities notably related to the antiviral responses (63).

\section{PHLEBOVIRUSES: RIFT VALLEY FEVER VIRUS}

Rift Valley Fever Virus (RVFV) is a mosquito-borne zoonotic pathogen that has caused large outbreaks in sub-Saharan countries, Yemen, Saudi Arabia, South Africa and Madagascar. RVFV infection is lethal for newborn animals and causes febrile illness and a high rate of abortion in adult ruminants. Humans infected with RVFV usually develop an acute febrile myalgic syndrome. However, in a small proportion of patients, RVFV infection leads to hepatic damage, hemorrhagic fever-like illness, encephalitis and/or retinal vasculitis that result in a lethal illness. The $\mathrm{S}$ segment utilizes an ambisense strategy and codes for the nucleoprotein $\mathrm{N}$ and the non-structural NSs protein in opposite polarities (65). This later protein is not essential for the viral replication. In addition, while the replication cycle occurs in the cytoplasm as it is the case for all bunyaviruses, NSs is located in the nucleus of infected cells, forming a filamentous structure that is unique among bunyaviruses (66). It was also demonstrated that the virulence was linked to the absence of IFN production due to NSs, which was shown to block transcription of the IFN $\beta$ $(67,68)$.

\subsection{The multifunctionality of the RVFV NSs protein}

At least, three complementary mechanisms linked to the interaction of NSs with three different cellular partners, enable RVFV to evade the innate antiviral host responses (Figure 4). These mechanisms target the expression of IFN- $\beta$, the activity of cellular transcription and translation.

The specific inhibition of IFN $\beta$ mRNA induction by NSs occurs as early as 3-4 h post-infection (p.i.). The molecular mechanism sustaining the transcriptional repressed state of the IFN $\beta$ promoter is correlated to the interaction between YY1 (the activator/repressor of IFN $\beta$ transcription Ying Yang 1), NSs and SAP30 (Sin3A Associated Protein 30) that is a subunit of Sin3A co-repressor complexes (such as NcoR/SMRT). It has been shown that SAP30 binds both NSs on and YY1 proteins, forming a complex that contains NcoR, HDAC1 and HDAC3, repressing the recruitment of CBP, the acetylation of histone H3 and consequently the transcriptional activation at the IFN $\beta$ promoter. To ascertain the role of this interaction, we created through a reverse genetic system, a recombinant ZH548 RVFV (ZH548-NSs $\Delta 210-230$ ) that expresses a mutated NSs protein unable to interact with SAP30. Such recombinant virus cannot inhibit the IFN production and is avirulent in mice (69).

Later during the viral cycle, after $8 \mathrm{~h}$ p.i., a second mechanism leads to the inhibition of the cellular RNA synthesis; it involves the interaction between NSs and the TFIIH factor. This complex is one of the basal transcription factors that can be resolved in two sub-complexes: the core that contains XPB, p62, p52, p44, p34 and TTD-A p8 is bridged by the XPD subunit to the CAK (Cdk- Activating Kinase) composed of cdk7, cyclin H, and MAT1 proteins. TFIIH possesses several enzymatic activities during transcription: (1) the XPB participates in promoter opening through a helicase/ATPase activity; (2) cdk7 phosphorylates RNA polymerase II (CTD-ser-5 residue) and numerous transcription factors controlling gene expression (70). The RVFV NSs protein suppresses the synthesis of host RNA by interacting and sequestrating p44 and XPB into the NSs nuclear filament. Through this interaction, NSs competes also with the usual partner of $\mathrm{p} 44$, XPD, unabling it to enter into the nucleus. The RVFV NSs protein also promotes the proteolysis of the TFIIH subunit p62 through the proteasome (71). Altogether, the effects of the NSs/p44 and NSs/p62 interactions inhibit the formation of TFIIH. Consequently, its concentration strongly decreases in RVFV infected cells leading to the inhibition of the cellular transcription (72).

A third function for NSs was recently described preventing the inhibition of host and viral translation $(31,73)$. RVFV is resistant to the PKR-mediated virus inhibition early in the course of the infection. Such a role is linked to the post-transcriptional down-regulation of PKR degraded through the proteasome and consequently the impairment of the phosphorylation of eIF $2 \alpha$.

In addition, it was shown that the formation of NSs filament strongly disturbs the architecture of nucleus of RVFVinfected cells and affects chromosome cohesion leading to segregation defects in murine and ovine cells. The genomic DNA is largely excluded from the NSs filament. However, specific DNA regions associated with heterochromatin can interact with NSs such as pericentromeric gamma-satellite sequences (74). Such a nuclear reorganization may be involved in the foetal deformities, necrosis of the placenta and abortions observed in infected ruminants. Although it seems likely that NSs proteins of different bunyaviruses have their specific partners $(31,69)$, the NSs proteins from other phleboviruses like Toscana (TOSV) and Punta Toro (PTV) viruses are also involved in the inhibition of IFN $\beta$-induction $(75,76)$, strongly indicating that NSs proteins have conserved functions through unique mode of actions.

\subsection{Anti-apoptotic role of RFVF NSm proteins}

A recent analysis of RVFV infection in cells of the lung epithelium indicated a viral strategy that aims at controlling

cellular apoptosis (77). Indeed, a recombinant RVFV lacking the expression of NSm proteins, encoded by the M segment, can 
induce extensive cell death. The expression of NSm, dispensable for viral replication, suppresses apoptosis triggered through the cleavage of caspase- 8 and -9 induced by staurospine (78). However, the control of apoptosis by RVFV seems complex since significant changes in PI3K-AKT, caspase, MAPK and p53 pathways, involved in survival and death of cells, were also observed (77).

\section{PERSPECTIVE}

Altogether the data on how bunyaviruses evade the cellular response indicate that each virus has developed its own strategy. For orthobunyaviruses and phleboviruses, NSs and to a lesser extend NSm proteins play a crucial role but in the case of hantaviruses, also other proteins such as the nucleocapsid and the glycoproteins appear to have IFN antagonist functions. For nairoviruses, less is known on the IFN antagonistic activities but the L polymerase may be the virulent factor. Such studies were important for the production of rationally designed attenuated vaccines, which have been developed so far, for RVFV. The naturally avirulent Clone 13 carrying a large deletion in the NSs ORF appears as a good vaccine candidate to protect against virulent RVFV (79). Other viruses obtained by reverse genetics were also produced with the deletion of the complete NSs ORF and /or the preglycoprotein region corresponding to $\operatorname{NSm}(33,78,80)$. Creation of mutated viruses by reverse genetics has been a valuable tool to decipher the function of the non-structural proteins $(21,23,69)$ but the methodology is not yet available for hantaviruses and nairoviruses. Other investigations are needed for the development of antivirals targeting the different steps of the viral cycle, which would be of great value for the treatment of these infections. Up to now, most of the studies focused on the viral functions antagonizing the innate immune response which is an immediate and the first line of defence. However, viruses have evolved other strategies to target cellular functions, which participate to the viral pathogenesis and will deserve to be studied in the near future.

\section{ACKNOWLEDGEMENTS}

N.L.M. is recipient from the ARC and a young investigator contract from the Institut National de la Santé et de la Recherche Médicale. The financial support from the Agence nationale de la Recherche (ANR-08-MIE-022) is greatly acknowledged.

\section{REFERENCES}

1. Kate Jones, Nikkita Patel, Marc Levy, Adam Storeygard, Deborah Balk, John Gittleman and Peter Daszak: Global trends in emerging infectious diseases. Nature, 451(7181), 990-3 (2008)

2. Richard Elliott: Bunyaviruses and climate change. Clin Microbiol Infect, 15(6), 510-7 (2009)

3. Ernie Gould and Stephen Higgs: Impact of climate change and other factors on emerging arbovirus diseases. Trans $R$ Soc Trop Med Hyg, 103(2), 109-21 (2009)

4. Yong-Zhen Zhang, Dun-Jin Zhou, Yanwen Xiong, Xiao-Ping Chen, Yong-Wen He, Qiangzheng Sun, Bin Yu, J. Li, Yong-An Dai, Jun-Hua Tian, Xin-Cheng Qin, Dong Jin, Zhigang Cui, Xue-Lian Luo, Wei Li, Shan Lu, Wen Wang, Jin-Song Peng, WenPing Guo, Ming-hui Li, Zhen-Jun Li, Shaomin Zhang, Chen Chen, Yan Wang, Menno de Jong and Jianguo Xu: [Hemorrhagic fever caused by a novel tick-borne Bunyavirus in Huaiyangshan, China.]. Zhonghua Liu Xing Bing Xue Za Zhi, 32(3), 209-220 (2011)

5. Xue-Jie Yu, Mi-Fang Liang, Shou-Yin Zhang, Yan Liu, Jian-Dong Li, Yu-Lan Sun, Lihong Zhang, Quan-Fu Zhang, Vsevolod Popov, Chuan Li, Jing Qu, Qun Li, Yan-Ping Zhang, Rong Hai, Wei Wu, Qin Wang, Fa-Xian Zhan, Xian-Jun Wang, Biao Kan, Shi-Wen Wang, Kang-Li Wan, Huai-Qi Jing, Jin-Xin Lu, Wen-Wu Yin, Hang Zhou, Xu-Hua Guan, Jia-Fa Liu, Zhen-Qiang Bi, Guo-Hua Liu, Jun Ren, Hua Wang, Zhuo Zhao, Jing-Dong Song, Jin-Rong He, Tao Wan, Jin-Shang Zhang, Xiao-Ping Fu, Li-Na Sun, Xiu-Ping Dong, Zi-Jian Feng, Wei-Zhong Yang, Tao Hong, Yu Zhang, David Walker, Yu Wang and De-Xin Li: Fever with thrombocytopenia associated with a novel bunyavirus in China. N Engl J Med, 364(16), 1523-32 (2011)

6. Richard Elliott, Michèle Bouloy, Charles Calisher, Rob Goldbach, Jack Moyer, Stuart Nichol, Ralf Pettersson, Alexander Plyusnin and Connie Schmaljohn: Family Bunyaviridae. In: Virus taxonomy. classification and nomenclature of viruses. Seventh report of the intenational committee on taxonomy of viruses. Ed M. H. V. Van Regenmortel, C. M. Fauquet, D. H. L. Bishop, E. B. Cartens, M. K. Estes, L. S. M., J. Maniloff, M. A. Mayo, D. J. McGeoch, P. C. R.\&W. R.B. academic press, San Diego (2000)

7. Mohammed Mir: Hantaviruses. Clin Lab Med, 30(1), 67-91 (2010)

8. Sonia Gerrard and Stuart Nichol: Characterization of the Golgi retention motif of Rift Valley fever virus G(N) glycoprotein. Journal of Virology, 76(23), 12200-10 (2002)

9. Xiaohong Shi, Joel van Mierlo, Andrew French and Richard Elliott: Visualizing the replication cycle of bunyamwera orthobunyavirus expressing fluorescent protein-tagged Gc glycoprotein. $J$ Virol, 84(17), 8460-9 (2010) 
10. Juan Reguera, Friedemann Weber and Stephen Cusack: Bunyaviridae RNA polymerases (L-protein) have an N-terminal, influenza-like endonuclease domain, essential for viral cap-dependent transcription. PLoS Pathog, 6(9) (2010)

11. Shuenn-Jue Wu, Geraldine Grouard-Vogel, Wellington Sun, John Mascola, Elena Brachtel, Ravitaht Putvatana, Mark Louder, Luis Filgueira, Mary Marovich, Henry Wong, Andrew Blauvelt, Gerald Murphy, Merlin Robb, Bruce Innes, Deborah Birx, Curtis Hayes and Sarah Frankel: Human skin Langerhans cells are targets of dengue virus infection. Nat Med, 6(7), 816-20 (2000)

12. Kristin Rogers and Mark Heise: Modulation of cellular tropism and innate antiviral response by viral glycans. $J$ Innate Immun, 1(5), 405-12 (2009)

13. Alick Isaacs and Jean Lindenmann: Virus interference. I. The interferon. Proc R Soc Lond B Biol Sci, 147(927), 258-67 (1957)

14. Alick Isaacs, Jean Lindenmann and Roger Valentine: Virus interference. II. Some properties of interferon. Proc R Soc Lond B Biol Sci, 147(927), 268-73 (1957)

15. Katherine Fitzgerald, Sarah McWhirter, Kerrie Faia, Daniel Rowe, Eicke Latz, Douglas Golenbock, Anthony Coyle, Sha-Mei Liao and Tom Maniatis: IKKepsilon and TBK1 are essential components of the IRF3 signaling pathway. Nat Immunol, 4(5), 491$6(2003)$

16. Mayra Solis, Delphine Goubau, Raphaelle Romieu-Mourez, Pierre Genin, Ahmet Civas and John Hiscott: Distinct functions of IRF-3 and IRF-7 in IFN-alpha gene regulation and control of anti-tumor activity in primary macrophages. Biochem Pharmacol, 72(11), 1469-76 (2006)

17. Leonidas Platanias: Mechanisms of type-I- and type-II-interferon-mediated signalling. Nat Rev Immunol, 5(5), 375-86 (2005)

18. Christian Schindler, David Levy and Thomas Decker: JAK-STAT signaling: from interferons to cytokines. $J$ Biol Chem, 282(28), 20059-63 (2007)

19. Sandy Der, Aimin Zhou, Bryan Williams and Robert Silverman: Identification of genes differentially regulated by interferon alpha, beta, or gamma using oligonucleotide arrays. Proc Natl Acad Sci U S A, 95(26), 15623-8 (1998)

20. Ganes Sen and Gregory Peters: Viral stress-inducible genes. Adv Virus Res, 70, 233-63 (2007)

21. Gjon Blakqori, Sophie Delhaye, Matthias Habjan, Carol Blair, Irma Sanchez-Vargas, Ken Olson, Ghassem Attarzadeh-Yazdi, Rennos Fragkoudis, Alain Kohl, Ulrich Kalinke, Siegfried Weiss, Thomas Michiels, Peter Staeheli and Friedemann Weber: La Crosse bunyavirus nonstructural protein NSs serves to suppress the type I interferon system of mammalian hosts. J Virol, 81(10), 4991-9 (2007)

22. Friedemann Weber, Anne Bridgen, John Fazakerley, Hein Streitenfeld, Nina Kessler, Richard Randall and Richard Elliott: Bunyamwera bunyavirus nonstructural protein NSs counteracts the induction of alpha/beta interferon. J Virol, 76(16), 7949-55 (2002)

23. Anne Bridgen, Friedemann Weber, John Fazakerley and Richard Elliott: Bunyamwera bunyavirus nonstructural protein NSs is a nonessential gene product that contributes to viral pathogenesis. Proc Natl Acad Sci U S A, 98(2), 664-9 (2001)

24. Hein Streitenfeld, Amanda Boyd, John Fazakerley, Anne Bridgen, Richard Elliott and Friedemann Weber: Activation of PKR by Bunyamwera virus is independent of the viral interferon antagonist NSs. J Virol, 77(9), 5507-11 (2003)

25. Alain Kohl, Reginald Clayton, Friedemann Weber, Anne Bridgen, Richard Randall and Richard Elliott: Bunyamwera virus nonstructural protein NSs counteracts interferon regulatory factor 3-mediated induction of early cell death. J Virol, 77(14), 79998008 (2003)

26. Daniel Thomas, Gjon Blakqori, Valentina Wagner, Marius Banholzer, Nina Kessler, Richard Elliott, Otto Haller and Friedemann Weber: Inhibition of RNA polymerase II phosphorylation by a viral interferon antagonist. J Biol Chem, 279(30), 31471-7 (2004)

27. Vincent Leonard, Alain Kohl, Thimothy Hart and Richard Elliott: Interaction of Bunyamwera Orthobunyavirus NSs protein with mediator protein MED8: a mechanism for inhibiting the interferon response. J Virol, 80(19), 9667-75 (2006) 
28. Amelia Casamassimi and Claudio Napoli: Mediator complexes and eukaryotic transcription regulation: an overview. Biochimie, 89(12), 1439-46 (2007)

29. Zhiyuan Yang, Jasper Yik, Ruichan Chen, Nanhai He, Moon Kyoo Jang, Keiko Ozato and Qiang Zhou: Recruitment of P$\mathrm{TEFb}$ for stimulation of transcriptional elongation by the bromodomain protein Brd4. Mol Cell, 19(4), 535-45 (2005)

30. Ingeborg van Knippenberg, Charlie Carlton-Smith and Richard Elliott: The N-terminus of Bunyamwera orthobunyavirus NSs protein is essential for interferon antagonism. J Gen Virol, 91(Pt 8), 2002-6 (2010)

31. Matthias Habjan, Andreas Pichlmair, Richard Elliott, Anna Overby, Timo Glatter, Matthias Gstaiger, Giulio Superti-Furga, Hermann Unger and Friedemann Weber: NSs protein of rift valley fever virus induces the specific degradation of the doublestranded RNA-dependent protein kinase. J Virol, 83(9), 4365-75 (2009)

32. Paul Verbruggen, Marius Ruf, Gjon Blakqori, Anna Overby, Martin Heidemann, Dirk Eick and Friedemann Weber: Interferon antagonist NSs of La Crosse virus triggers a DNA damage response-like degradation of transcribing RNA polymerase II. J Biol Chem, 286(5), 3681-92 (2011)

33. Gjon Blakqori and Friedemann Weber: Efficient cDNA-based rescue of La Crosse bunyaviruses expressing or lacking the nonstructural protein NSs. J Virol, 79(16), 10420-8 (2005)

34. Daniel Colon-Ramos, Pablo Irusta, Eugene Gan, Michael Olson, Jaewhan Song, Richard Morimoto, Richard Elliott, Mark Lombard, Robert Hollingsworth, J Marie Hardwick, Gary Smith and Sally Kornbluth: Inhibition of translation and induction of apoptosis by Bunyaviral nonstructural proteins bearing sequence similarity to reaper. Mol Biol Cell, 14(10), 4162-72 (2003)

35. Samantha Soldan, Matthew Plassmeyer, Meghan Matukonis and Francisco Gonzalez-Scarano: La Crosse virus nonstructural protein NSs counteracts the effects of short interfering RNA. J Virol, 79(1), 234-44 (2005)

36. Jessica Levine, Joseph Prescott, Kyle Brown, Sonja Best, Hideki Ebihara and Heinz Feldmann: Antagonism of type I interferon responses by new world hantaviruses. $J$ Virol, 84(22), 11790-801 (2010)

37. Valery Matthys, Elena Gorbunova, Irina Gavrilovskaya, Timothy Pepini and Erich Mackow: The C-terminal 42 residues of the tula virus gn protein regulate interferon induction. J Virol, 85(10), 4752-60 (2011)

38. Peter Alff, Irina Gavrilovskaya, Elena Gorbunova, Karen Endriss, Yuson Chong, Erika Geimonen, Nandini Sen, Nancy Reich and Erich Mackow: The pathogenic NY-1 hantavirus G1 cytoplasmic tail inhibits RIG-I- and TBK-1-directed interferon responses. J Virol, 80(19), 9676-86 (2006)

39. Peter Alff, Nandini Sen, Elena Gorbunova, Irina Gavrilovskaya and Erich Mackow: The NY-1 hantavirus Gn cytoplasmic tail coprecipitates TRAF3 and inhibits cellular interferon responses by disrupting TBK1-TRAF3 complex formation. J Virol, 82(18), 9115-22 (2008)

40. Joel Pomerantz and David Baltimore: NF-kappaB activation by a signaling complex containing TRAF2, TANK and TBK1, a novel IKK-related kinase. EMBO J, 18(23), 6694-704 (1999)

41. Gagik Oganesyan, Supriya Saha, Beichu Guo, Jeannie He, Arash Shahangian, Brian Zarnegar, Andrea Perry and Genhong Cheng: Critical role of TRAF3 in the Toll-like receptor-dependent and -independent antiviral response. Nature, 439(7073), 20811 (2006)

42. Christina Spiropoulou, César Albarino, Thomas Ksiazek and Pierre Rollin: Andes and Prospect Hill hantaviruses differ in early induction of interferon although both can downregulate interferon signaling. J Virol, 81(6), 2769-76 (2007)

43. Mohammed Mir and Antonito Panganiban: Trimeric hantavirus nucleocapsid protein binds specifically to the viral RNA panhandle. J Virol, 78(15), 8281-8 (2004)

44. Mohammed Mir and Antonito Panganiban: The hantavirus nucleocapsid protein recognizes specific features of the viral RNA panhandle and is altered in conformation upon RNA binding. $J$ Virol, 79(3), 1824-35 (2005)

45. Erdong Cheng, Absarul Haque, Mary Ashley Rimmer, Islam Hussein, Sheema Sheema, Alex Little and Mohammed Mir: Characterization of the Interaction between Hantavirus Nucleocapsid Protein (N) and Ribosomal Protein S19 (RPS19). J Biol Chem, 286(13), 11814-24 (2011) 
46. Byoung-Hee Lee, Kumiko Yoshimatsu, Aakihiko Maeda, Kazuhiko Ochiai, Masami Morimatsu, Koichi Araki, Michiko Ogino, Shigeru Morikawa and Jiro Arikawa: Association of the nucleocapsid protein of the Seoul and Hantaan hantaviruses with small ubiquitin-like modifier-1-related molecules. Virus Res, 98(1), 83-91 (2003)

47. Pasi Kaukinen, Antti Vaheri and Alexander Plyusnin: Non-covalent interaction between nucleocapsid protein of Tula hantavirus and small ubiquitin-related modifier-1, SUMO-1. Virus Res, 92(1), 37-45 (2003)

48. Xiao-Dong Li, Tomi Makela, Deyin Guo, Rabah Soliymani, Vesa Koistinen, Olli Vapalahti, Antti Vaheri and Hilkka Lankinen: Hantavirus nucleocapsid protein interacts with the Fas-mediated apoptosis enhancer Daxx. J Gen Virol, 83(Pt 4), 759$66(2002)$

49. Shannon Taylor, Natalia Frias-Staheli, Adolfo Garcia-Sastre and Connie Schmaljohn: Hantaan virus nucleocapsid protein binds to importin alpha proteins and inhibits tumor necrosis factor alpha-induced activation of nuclear factor kappa B. $J$ Virol, 83(3), 1271-9 (2009)

50. Shannon Taylor, Ryan Krempel and Connie Schmaljohn: Inhibition of TNF-alpha-induced activation of NF-kappaB by hantavirus nucleocapsid proteins. Ann N Y Acad Sci, 1171 Suppl 1, E86-93 (2009)

51. Alexander Plyusnin: Genetics of hantaviruses: implications to taxonomy. Arch Virol, 147(4), 665-82 (2002)

52. Kirsi Jaaskelainen, Angelina Plyusnina, Aake Lundkvist, Antti Vaheri and Alexander Plyusnin: Tula hantavirus isolate with the full-length ORF for nonstructural protein NSs survives for more consequent passages in interferon-competent cells than the isolate having truncated NSs ORF. Virol J, 5, 3 (2008)

53. Jussi Virtanen, Kirsi Jaaskelainen, J. Djupsjobacka, Antti Vaheri and Alexander Plyusnin: Tula hantavirus NSs protein accumulates in the perinuclear area in infected and transfected cells. Arch Virol, 155(1), 117-21 (2010)

54. Kirsi Jaaskelainen, Pasi Kaukinen, Ekaterina Minskaya, Angelina Plyusnina, Olli Vapalahti, Richard Elliott, Friedemann Weber, Antti Vaheri and Alexander Plyusnin: Tula and Puumala hantavirus NSs ORFs are functional and the products inhibit activation of the interferon-beta promoter. J Med Virol, 79(10), 1527-36 (2007)

55. Alexander Plyusnin and Sergey Morzunov: Virus evolution and genetic diversity of hantaviruses and their rodent hosts. Curr Top Microbiol Immunol, 256, 47-75 (2001)

56. Friedemann Weber and Ali Mirazimi: Interferon and cytokine responses to Crimean Congo hemorrhagic fever virus; an emerging and neglected viral zonoosis. Cytokine Growth Factor Rev, 19(5-6), 395-404 (2008)

57. Sandor Bereczky, Gunnel Lindegren, Helen Karlberg, Sara Akerstrom, Jonas Klingstrom and Ali Mirazimi: Crimean-Congo hemorrhagic fever virus infection is lethal for adult type I interferon receptor-knockout mice. J Gen Virol, 91(Pt 6), 1473-7 (2010)

58. Dennis Bente, Judie Alimonti, Wun-Ju Shieh, Gaelle Camus, Ute Stroher, Sherif Zaki and Steven Jones: Pathogenesis and immune response of Crimean-Congo hemorrhagic fever virus in a STAT-1 knockout mouse model. J Virol, 84(21), 11089-100 (2010)

59. Ida Andersson, Linda Bladh, Mehrdad Mousavi-Jazi, Karl-Eric Magnusson, Ake Lundkvist, Otto Haller and Ali Mirazimi: Human MxA protein inhibits the replication of Crimean-Congo hemorrhagic fever virus. J Virol, 78(8), 4323-9 (2004)

60. Ida Andersson, Ake Lundkvist, Otto Haller and Ali Mirazimi: Type I interferon inhibits Crimean-Congo hemorrhagic fever virus in human target cells. J Med Virol, 78(2), 216-22 (2006)

61. Matthias Habjan, Ida Andersson, Jonas Klingstrom, Michael Schumann, Aarnold Martin, Petra Zimmermann, Valentina Wagner, Andreas Pichlmair, Urs Schneider, Elke Muhlberger, Ali Mirazimi and Friedemann Weber: Processing of genome 5' termini as a strategy of negative-strand RNA viruses to avoid RIG-I-dependent interferon induction. PLoS One, 3(4), e2032 (2008)

62. Ida Andersson, Helen Karlberg, Mehrdad Mousavi-Jazi, Luis Martinez-Sobrido, Friedemann Weber and Ali Mirazimi: Crimean-Congo hemorrhagic fever virus delays activation of the innate immune response. J Med Virol, 80(8), 1397-404 (2008)

63. Natalia Frias-Staheli, Nadia Giannakopoulos, Marjolein Kikkert, Shannon Taylor, Anne Bridgen, Jason Paragas, Juergen Richt, Raymond Rowland, Connie Schmaljohn, Deborah Lenschow, Eric Snijder, Adolfo Garcia-Sastre and Herbert Virgin: 
Ovarian tumor domain-containing viral proteases evade ubiquitin- and ISG15-dependent innate immune responses. Cell Host Microbe, 2(6), 404-16 (2007)

64. Eric Bergeron, Cesar Albarino, Marina Khristova and Stuart Nichol: Crimean-Congo hemorrhagic fever virus-encoded ovarian tumor protease activity is dispensable for virus RNA polymerase function. J Virol, 84(1), 216-26 (2010)

65. Colomba Giorgi, Luisa Accardi, Loredana Nicoletti, Maria Cristina Gro, Kazuhiko Takehara, C Hilditch, Saeko Morikawa and David Bishop: Sequences and coding strategies of the S RNAs of Toscana and Rift Valley fever viruses compared to those of Punta Toro, Sicilian Sandfly fever, and Uukuniemi viruses. Virology, 180(2), 738-53 (1991)

66. Ramon Flick and Michèle Bouloy: Rift Valley fever virus. Curr Mol Med, 5(8), 827-34 (2005)

67. Agnès Billecocq, Martin Spiegel, Pierre Vialat, Alain Kohl, Friedemann Weber, Michèle Bouloy and Otto Haller: NSs protein of Rift Valley fever virus blocks interferon production by inhibiting host gene transcription. J Virol, 78(18), 9798-806 (2004)

68. Michèle Bouloy, Christian Janzen, Pierre Vialat, Huot Khun, Jovan Pavlovic, Michel Huerre and Otto Haller: Genetic evidence for an interferon-antagonistic function of rift valley fever virus nonstructural protein NSs. J Virol, 75(3), 1371-7 (2001)

69. Nicolas Le May, Zeyni Mansuroglu, Psylvia Leger, Thibaut Josse, Guillaume Blot, Agnès Billecocq, Ramon Flick, Yves Jacob, Eliette Bonnefoy and Michèle Bouloy: A SAP30 complex inhibits IFN-beta expression in Rift Valley fever virus infected cells. PLoS Pathog, 4(1), e13 (2008)

70. Alexander Zhovmer, Valentin Oksenych and Fréderic Coin: Two sides of the same coin: TFIIH complexes in transcription and DNA repair. ScientificWorldJournal, 10, 633-43 (2010)

71. Birte Kalveram, Olga Lihoradova and Tetsuro Ikegami: NSs Protein of Rift Valley Fever Virus Promotes Post-Translational Downregulation of the TFIIH Subunit p62. J Virol (2011)

72. Nicolas Le May, Sandy Dubaele, Luca Proietti De Santis, Agnès Billecocq, Michèle Bouloy and Jean-Marc Egly: TFIIH transcription factor, a target for the Rift Valley hemorrhagic fever virus. Cell, 116(4), 541-50 (2004)

73. Tetsuro Ikegami, Krishna Narayanan, Sungyong Won, Wataru Kamitani, C. J. Peters and Shinji Makino: Rift Valley fever virus NSs protein promotes post-transcriptional downregulation of protein kinase PKR and inhibits eIF2alpha phosphorylation. PLoS Pathog, 5(2), e1000287 (2009)

74. Zeyni Mansuroglu, Tibaut. Josse, Jérome Gilleron, Agnès Billecocq, Psylvia Leger, Michèle Bouloy and Eliette Bonnefoy: Nonstructural NSs protein of rift valley fever virus interacts with pericentromeric DNA sequences of the host cell, inducing chromosome cohesion and segregation defects. J Virol, 84(2), 928-39 (2010)

75. Lucy Perrone, Krishna Narayanan, Melissa Worthy and C. J. Peters: The S segment of Punta Toro virus (Bunyaviridae, Phlebovirus) is a major determinant of lethality in the Syrian hamster and codes for a type I interferon antagonist. $J$ Virol, 81(2), 884-92 (2007)

76. Gianni Gori Savellini, Friedemann Weber, Chiara Terrosi, Matthias Habjan, Barbara Martorelli and Maria Grazia Cusi: Toscana virus induces interferon although its NSs protein reveals antagonistic activity. J Gen Virol, 92(Pt 1), 71-9 (2011)

77. Taissia Popova, Michael Turell, Virginia Espina, Kylene Kehn-Hall, Jessica Kidd, Aarthi Narayanan, Lance Liotta, Emmanuel Petricoin, 3rd, Fata Kashanchi, Charles Bailey and Serguei Popov: Reverse-phase phosphoproteome analysis of signaling pathways induced by Rift valley fever virus in human small airway epithelial cells. PLoS One, 5(11), e13805 (2010)

78. Sungyong Won, Tetsuro Ikegami, C. J. Peters and Shinji Makino: NSm protein of Rift Valley fever virus suppresses virusinduced apoptosis. J Virol, 81(24), 13335-45 (2007)

79. Baptiste Dungu, Ian Louw, Alison Lubisi, Pamela Hunter, Beate von Teichman and Michèle Bouloy: Evaluation of the efficacy and safety of the Rift Valley Fever Clone 13 vaccine in sheep. Vaccine, 28(29), 4581-7 (2010)

80. Tetsuro Ikegami, Sungyong Won, C. J. Peters and Shinji Makino: Rescue of infectious rift valley fever virus entirely from cDNA, analysis of virus lacking the NSs gene, and expression of a foreign gene. $J$ Virol, 80(6), 2933-40 (2006) 
Abbreviations: CHIKV: Chikungunya Virus, DENV: Dengue Virus, YFV: Yellow Fever Virus, CCHF: Crimea-Congo Hemorrhagic Fever Virus, RVFV: Rift Valley Fever Virus, UUKV: Uukuniemi Virus, L: Large, M: Medium, S: Small, L-RdRP: viral RNA-dependent RNA polymerase, RNPs: ribonucleoparticles, IFN: Interferon, DC: Dendritic Cell, pDC: plasmacytoid Dendritic Cell, PRR: Pattern Recognition Receptors, PAMP: Pathogen-Associated Molecular Patterns, ds: double-stranded, ss: single-stranded, PKR: Protein Kinase R, IRF-3: Interferon Regulatory Factor 3, mRNA: messenger RNA, eIF-2 $\alpha$ : eukaryotic Initiation Factor 2 alpha, IFNAR: Type I IFN receptor, JAK: Janus Kinase, STAT: Signal Transducer and Activator of Transcription, ISGF-3: IFN stimulated gene factor 3, ISRE: IFN-stimulated response elements, ISGs: IFN-stimulated genes, ADAR1: adenosine deaminase 1, BUNV: Bunyamwera Virus, LACV: La Crosse Virus, RNAPII: RNA polymerase II, ANDV: Andes Virus, SNV: Sin Nombre Virus, NY-1V: New York Virus 1, HNTV: Hantaan Virus, PUUV: Puumala Virus, SEOV: Seoul Virus, HCPS: Hantavirus Cardiopulmonary Syndrome, HFRS: Hemorrhagic Fever with Renal Syndrome, GPC: Glycoprotein Precursor, RPS19: Ribosomal Protein S19, Ubc9: Ubiquitin-conjugating enzyme 9, SUMO-1: the Small Ubiquitinlike Modifier 1, ORF: Open Reading Frame, OTU: ovarian tumor, ISG15: IFN-stimulated gene product 15, Ub: Ubiquitin, TULV: Tula Virus, PHV: Prospect Hill Virus, DUGV: Dugbe Virus, NSD: Nairobi Sheep Disease, SAP30: Sin3A associated protein, CAK: Cdk-Activating Kinase, TOSV: Toscana Virus, PTV: Punta Toro Virus, YY1: Ying Yang 1.

Key Words bunyaviruses, innate immunity, escape, virulence factors

Send correspondence to: Nicolas Le May, Institut de Génétique et de Biologie Moléculaire et Cellulaire, CNRS/INSERM/ULP, BP 163, 67404 Illkirch Cedex, C. U. Strasbourg, France, Tel: 33388653451, Fax: 33388653201, E-mail: nlemay@igbmc.fr

Table 1. Human pathogens in the different genera of the Bunyaviridae family

\begin{tabular}{|c|c|c|c|}
\hline Genus & Virus & Vector & Human Disease \\
\hline \multicolumn{4}{|c|}{ Orthobunyavirus } \\
\hline & La Crosse & Mosquito & Severe encephalitis, meningitis \\
\hline & Ngari & Mosquito & Haemorrhagic Fever \\
\hline & Oropouche & Midge & Acute febrile illness \\
\hline & Tahyna & Mosquito & Non fatal flu-like illness \\
\hline \multicolumn{4}{|c|}{ Hantavirus } \\
\hline & Andes & Mouse & $\begin{array}{l}\text { Hantavirus cardiopulmonary syndrome } \\
\text { Fatality } 40-50 \%\end{array}$ \\
\hline & Hantaan & Field Mouse & $\begin{array}{l}\text { Severe haemorrhagic fever with renal syndrome } \\
\text { Fatality } 5-15 \%\end{array}$ \\
\hline & Puumala & Bank Vole & $\begin{array}{l}\text { Mild haemorrhagic fever with renal syndrome } \\
\text { Fatality } 0.1 \%\end{array}$ \\
\hline & Seoul & Rat & $\begin{array}{l}\text { Moderate haemorrhagic fever with renal syndrome } \\
\text { Fatality } 1 \%\end{array}$ \\
\hline & Sin Nombre & Deer Mouse & $\begin{array}{l}\text { Hantavirus cardiopulmonary syndrome } \\
\text { Fatality } 50 \%\end{array}$ \\
\hline \multicolumn{4}{|c|}{ Nairovirus } \\
\hline & Crimean Congo hemorrhagic Fever & Tick & $\begin{array}{l}\text { Haemorrhagic Fever } \\
\text { Fatality } 20-80 \%\end{array}$ \\
\hline \multicolumn{4}{|c|}{ Phlebovirus } \\
\hline & Naples sandfly Fever & Sandfly & "three day fever" with Influenza-like symptoms \\
\hline & Punta Toro & Sandfly & Acute febrile illness \\
\hline & Rift Valley Fever & Mosquito & $\begin{array}{l}\text { Hepatitis, encephalitis, haemorrhagic fever } \\
\text { Fatality } 1-10 \%\end{array}$ \\
\hline & Toscana & Sandfly & Meningitis, meningoencephalitis, encephalitis \\
\hline & Sicilian sandfly Fever & Sandfly & "three day fever" with Influenza-like symptoms \\
\hline & Huaiyangshan & Tick & $\begin{array}{l}\text { severe thrombocytosis and multiorgan dysfunction } \\
\text { Fatality } 30 \%\end{array}$ \\
\hline
\end{tabular}

Figure 1. Induction and signaling pathways of the innate immunity. The viral replication of bunyaviruses can produce 5 , triphosphate ssRNA and dsRNA that activate the cytoplasmic PAMPs RIG-I, MDA-5 and PKR. The RIG-I/MDA-5 dependent pathways induce the mitochondrial MAVS (IPS-1/Cardiff/VISA) that is necessary for the subsequent TBK-1-directed phosphorylation of IRF-3 and NF-KB that translocate into the nucleus and transactivate collectively the IFN $\beta$ and IFN $\alpha 1$ promoters. In parallel, activated PKR can phosphorylate eIF2 $\alpha$ and inhibit the host and viral translation. The secreted IFN $\beta$ and IFN $\alpha 1$ acting in autocrine and paracrine manner bind and activate the IFNARs allowing the expression of numerous ISGs and IRF-7 through the JAK/STAT pathways. In these cells, bunyaviruses can be blocked through the products of these ISGs. Moreover, 5' triphosphate ssRNA and dsRNA produced during the viral replication activate the cytoplasmic RIG-I, MDA-5 and consequently IRF-7/IRF-3 leading to a positive-feedback loop that initiates the synthesis of IFN $\beta$ and several IFN $\alpha$ subtypes.

Figure 2. Mechanisms of immune escape targeting the cellular transcriptional machinery for BUNV, LACV and RVFV. NSs proteins from BUNV, LACV and RVFV block the type I IFN response and shut off the mRNA expression by targeting the cellular transcriptional machinery through different strategies. RVFV NSs protein inhibit the formation of the basal transcription TFIIH complex that is crucial for the initiation step by phosphorylating notably the CTD repeat serine 5 residue of RNAPII. BUNV and LACV NSs proteins induce the proteolysis of RNAPII engaged in elongation (characterized by the CTD repeat serine 


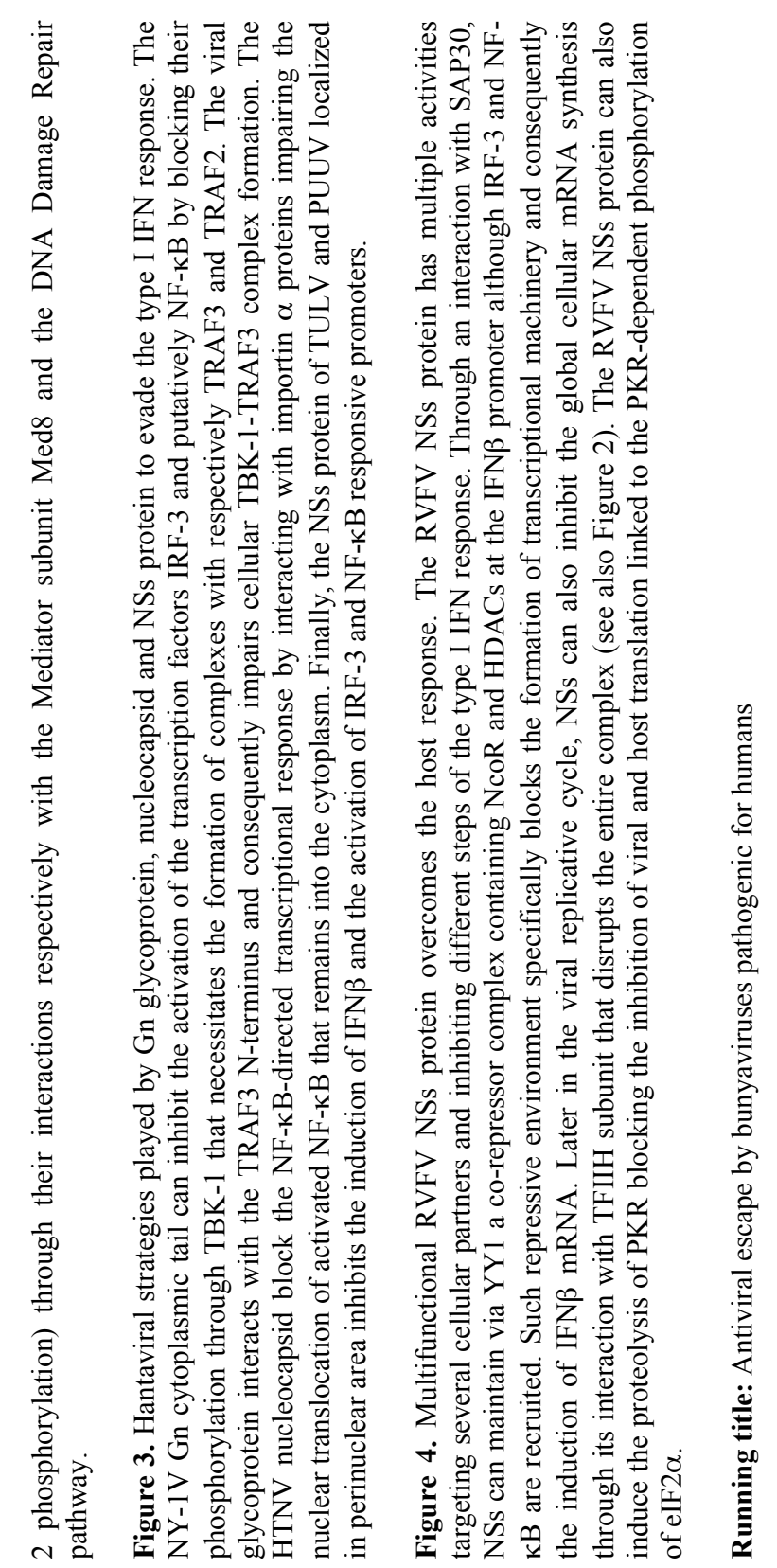




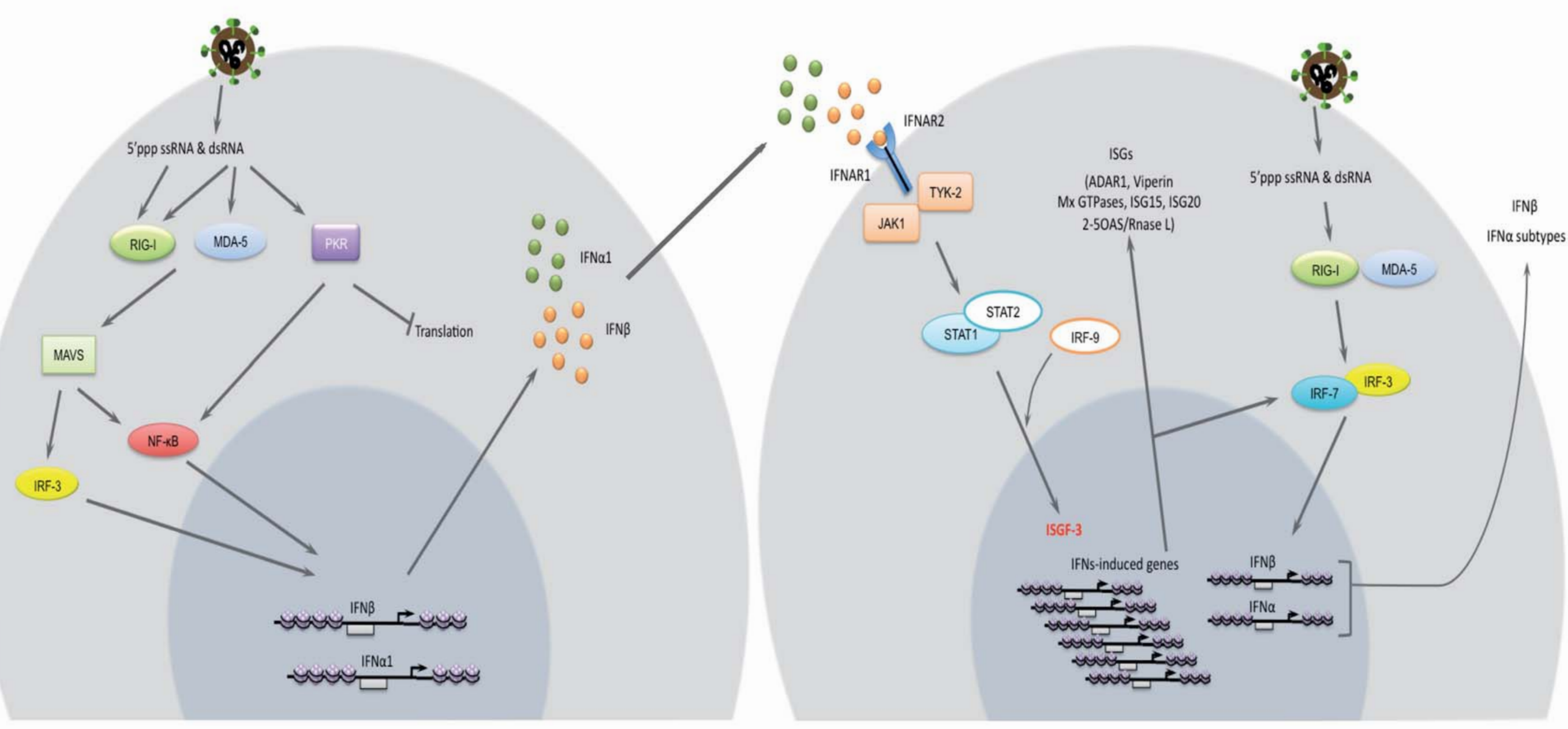

Figure 1 


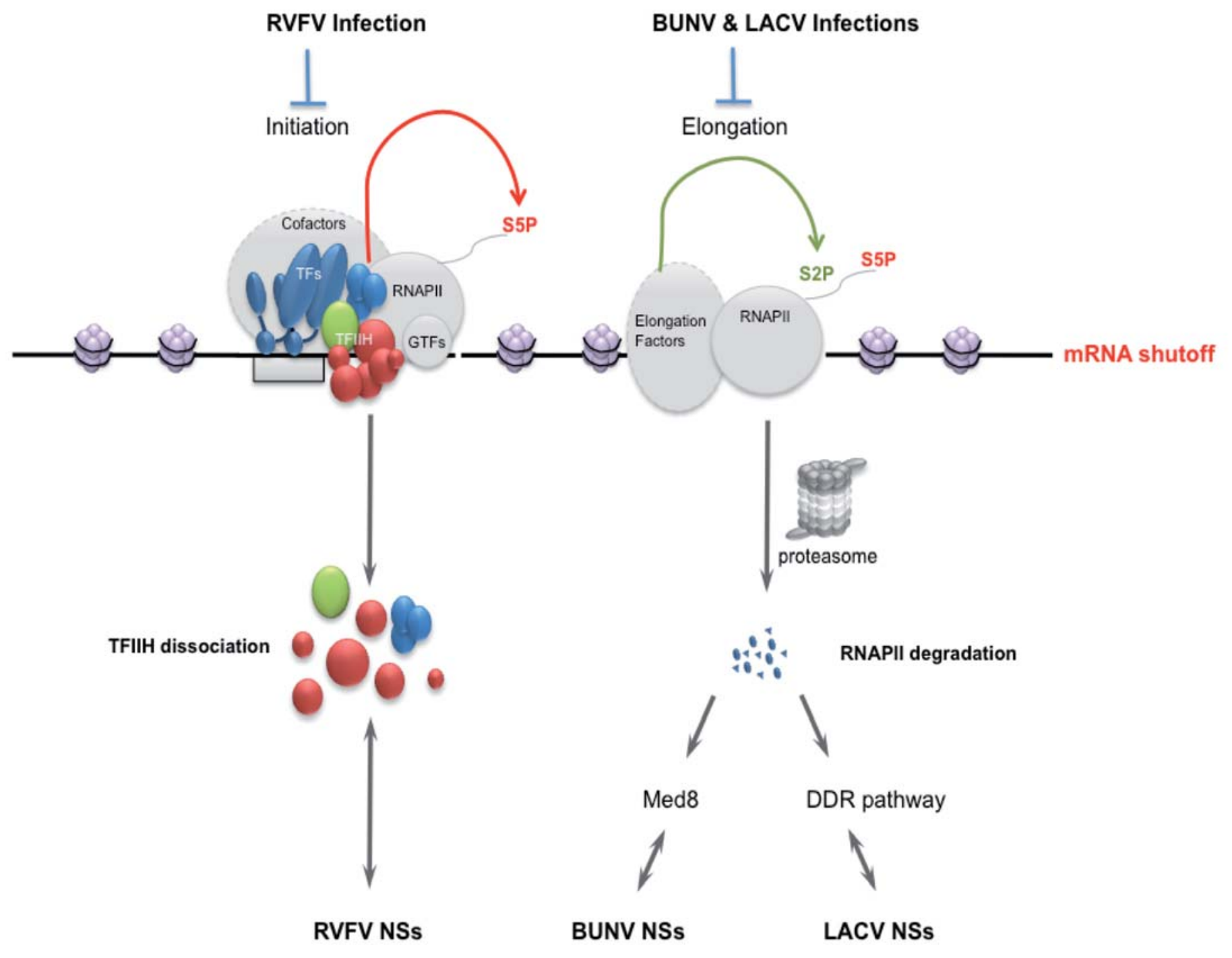




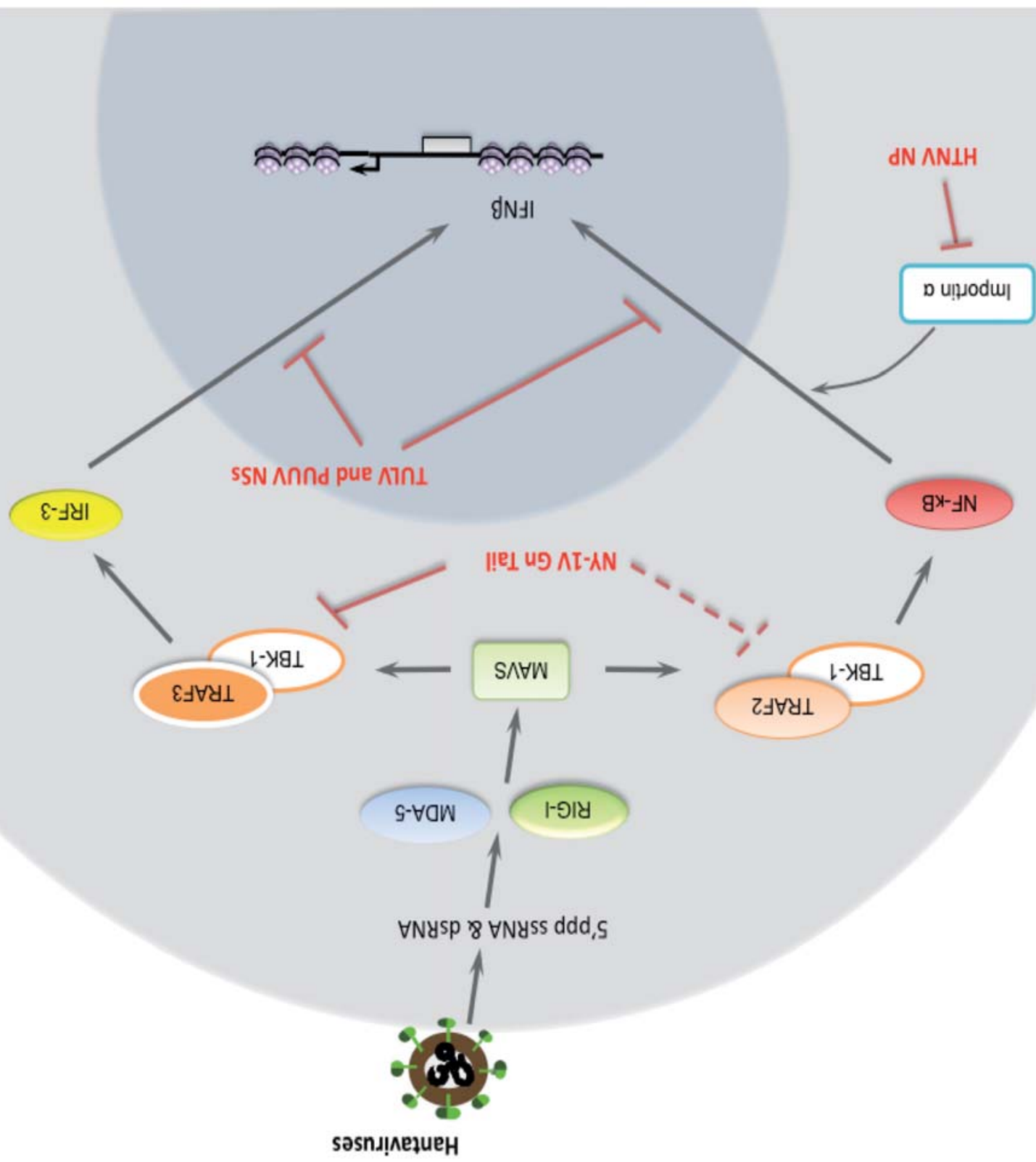




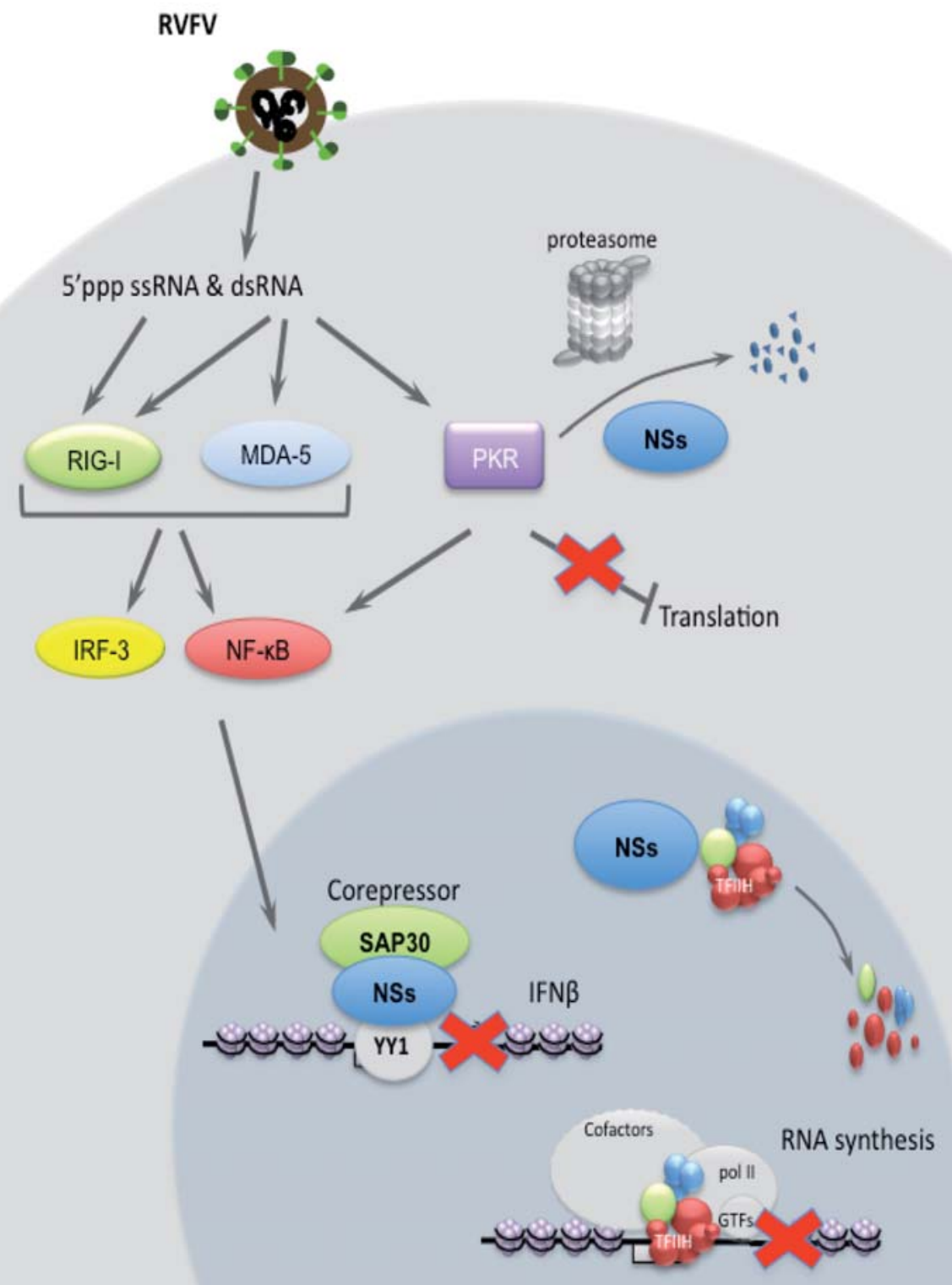

Figure 4 